\title{
Paint by number: Uncovering phase flows of an integrable dynamical system
}

\author{
Liam Healy and Etienne Deprit \\ Naval Center for Space Technology, Code 8242, Naval Research Laboratory, Washington, DC 20375
}

(Received 17 September 1990; accepted 18 February 1991)

Given an integrable dynamical system with one degree of freedom, "painting" the integral over phase space proves to be a powerful technique for uncovering both global and local behavior.This graphical technique avoids numerical integration, employing instead a nonlinear method of assigning contrasting colors to the energy values to distinguish subtle details of the flow.

\section{INTRODUCTION}

In this paper, we develop a graphical method of visualizing the global phase flow in certain Hamiltonian dynamics problems. We illustrate this method with the problem of an earth-orbiting satellite without drag. ${ }^{1}$ In the zonal harmonic model of this problem, the earth's gravitational field is expanded as a sum of Legendre polynomials $P_{k}$ with small coefficients $J_{k}$ and thus contains no longitudinal effects. The resultant Hamiltonian has two degrees of freedom: the radius vector to the satellite $r$, the polar angle $\theta$, and their conjugate momenta $R$ and $\Theta$,

$\mathscr{H}=\frac{1}{2}\left(R^{2}+\frac{\Theta^{2}}{r^{2}}\right)-\frac{\mu}{r}\left(1-\sum_{k>2}\left(\frac{\alpha}{r}\right)^{k} J_{k} P_{k}(\sin \chi)\right)$.

The latitude is given by $\chi$; the parameters $\mu$ and $\alpha$ are, respectively, the Keplerian constant and the equatorial radius of the earth.

In order to extract the long-term dynamics, we must put the Hamiltonian in normal form. ${ }^{2}$ This removes short period terms on the time scale of one orbital revolution, yielding a Hamiltonian accounting for long period effects. In the zonal problem of artificial satellite theory, the normalization reduces the dynamical system to one degree of freedom - the canonical variables being the argument of perigee $g$ and its conjugate momentum $G$, the norm of the angular momentum. Topologically, the phase space is a sphere. ${ }^{3}$ Points at the north pole of the phase sphere represent circular orbits for satellites; the south pole corresponds to satellites in equatorial orbit around the earth. The angle $g$ and the momentum $G$ form a system of cylindrical coordinates on the phase sphere.

The normalization proceeds order by order in the small quantities $J_{n}$ by means of a Lie transformation. ${ }^{4}$ Such a computation involves an exceptionally large amount of algebra which, by necessity, is performed with specialized computer algebra packages. ${ }^{5}$ Although often quite lengthy, the final result is far more compact than the intermediate formulas. In the so-called main problem where we retain only the lowest zonal harmonic $J_{2}$, we produced the normalized Hamiltonian:

$$
\begin{aligned}
\mathscr{H}^{*}= & -\frac{1}{2} n^{2} a^{2}+n G(\alpha / p)^{2}\left(\frac{1}{2}-\frac{3}{4} s^{2}\right)+\frac{1}{2} n G J_{2}^{2}(\alpha / p)^{4} \\
& \times\left\{-\frac{15}{8}+\frac{15}{4} s^{2}-\frac{105}{64} s^{4}+\eta\left(-\frac{3}{4}+\frac{9}{4} s^{2}-\frac{27}{16} s^{4}\right)\right. \\
& +\eta^{2}\left(\frac{3}{8}-\frac{3}{8} s^{2}-\frac{15}{64} s^{4}\right)+\cos 2 g\left[\frac{93}{16} s^{2}-\frac{225}{32} s^{4}\right. \\
& \left.\left.+(\beta+\eta)\left(-3 s^{2}+\frac{15}{4} s^{4}\right)+\eta^{2}\left(-\frac{21}{16} s^{2}+\frac{45}{32} s^{4}\right)\right]\right\} .
\end{aligned}
$$

For the meaning of the intermediate quantities $n, a, p, \beta, \eta$, and $s$, the reader is referred to Deprit. ${ }^{6}$ These Hamiltonians are often too complicated to extract analytically information about the global dynamics of the system. Thus we must resort to other methods.

\section{EXPLORING PHASE SPAGE}

Foremost among the features we want to uncover in phase space are the equilibria (in the problem at hand, these represent satellites traveling on fixed orbits) and the flow in their neighborhoods. At a deeper level, we want to know about the stability of critical points and, consequently, about the structure of the global phase flow. Finally, we want to understand how the phase space varies with the parameters of the system, such as an integral of the motion. When parameters vary, equilibria may bifurcate or coalesce, change position, or change stability.

One obvious approach to gaining this understanding is numerical integration of the equations of motion

$$
\dot{g}=\frac{\partial \mathscr{H}^{*}}{\partial G}, \quad \dot{G}=-\frac{\partial \mathscr{H}^{*}}{\partial g} .
$$


Numerical integration provides the information we are looking for, but at a cost. It is slow, particularly near equilibria, but these are precisely the points in which we are interested. Furthermore, as the normalized Hamiltonian grows more complex than Eq. (2) with the inclusion of higher zonal harmonics, the corresponding differential equations grow increasingly difficult to integrate, both in terms of time and precision.

Even more crucial in the use of numerical integration is the selection of initial conditions. Picking initial conditions that will reveal essential features of the phase space is quite difficult for, in a sense, it begs the question. Figure 1 shows a numerical integration for the main problem of satellite theory. Notice how the plot locates three stable equilibria at the center of the closed orbits, but leaves out much detail in detecting the two unstable equilibria above and below the central oval. Only the closed orbits surrounding the stable points lead us to conjecture the existence of unstable equilibria. This crude diagram required several minutes on a workstation-class machine for the integration, and several hours of experimentation to pick an optimal set of initial conditions.

Needless to say, searching for parametric bifurcation by numerical integration is an almost insufferable task. How can we rapidly obtain enough flow diagrams like Fig. 1 to make a movie revealing the system's evolution with changing parameters?

\section{PAINTING}

As an alternative to numerical integration, we propose to "paint" the phase space. Since $\mathscr{H} *$ is integrable with one degree of freedom, the level curves represent the orbits. We take advantage of this fact to sketch phase flows.

In essence, painting reduces to the following chain of mappings:

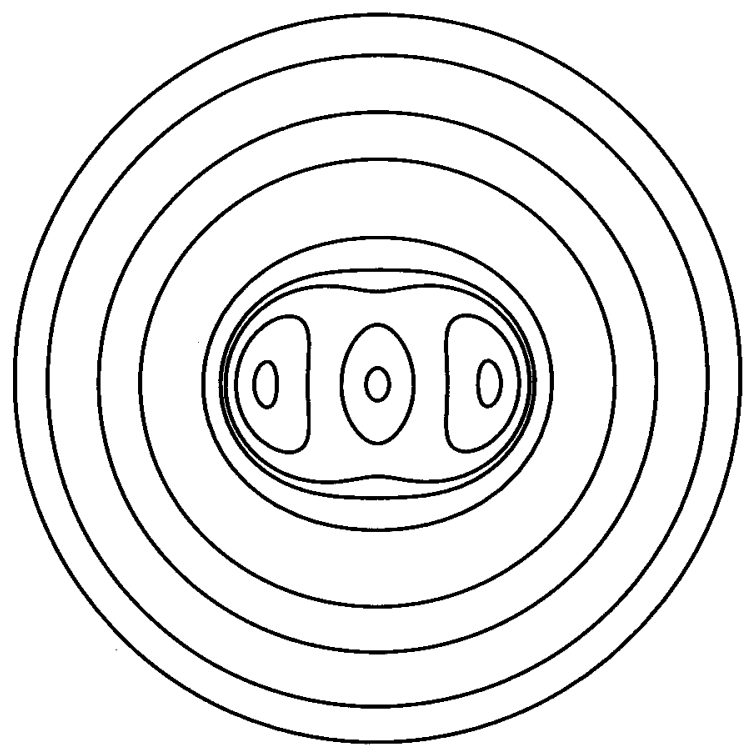

FIG. 1. Numerical integration of the northern hemisphere of phase space for the main problem of artificial satellite theory.

$$
\underset{S \rightarrow D \rightarrow R \rightarrow G \rightarrow C .}{\mathscr{P}} \stackrel{\mathscr{S}}{\mathscr{C}} \boldsymbol{B}
$$

The inverse projection $\mathscr{P}$ maps $p$ screen points $S$ into a subset of the phase space $D$. For instance, we might pick a view that covers all of the northern hemisphere for the spherical space in the satellite problem. The mapping $\mathscr{P}$ then takes all points in a circular disk on the screen to points on the hemisphere by an inverse orthographic projection, as shown in Fig. 2. This method simplifies the graphics algorithm by ensuring a one-to-one mapping of pixels to points on the hemisphere. This provides a simple way of rendering each pixel $p$ in $S$-we do not need to interpolate between energy values to color any pixels.

The Hamiltonian $\mathscr{H}^{*}$ maps points from phase space $D$ to energy values in the range $R$. Having found the energy values, we must then assign colors to the points of phase space. The grading function $\mathscr{G}$ then maps these function values into $G=[0, n)$ where $n$ is the number of grades. A grading function $\mathscr{G}$ is required to discretize the values in $R$ for display by the finite number of colors available on a graphics device; typical hardware provides $2^{8}$ or $2^{24}$ grades. The palette function $\mathscr{C}$ maps values from the grades $G$ into colors $C$.

By contrast with numerical integration, we do not compute the level curves of the Hamiltonian. In paintings of the phase space, level curves of $\mathscr{H}^{*}$ appear as lines of contiguous pixels with the same hue. The eye, not the processor, then connects the dots to reveal the orbits of the system. Obviously, finding detail in the image depends critically on how we choose the grading function $\mathscr{G}$ and the color palette $\mathscr{C}$. Sections III and IV offer choices that we found to be propitious.

\section{MAPPING VALUES IHTO GRADES}

In the problems we have examined, the critical points in phase space generally occur in wide plateaus - areas of low gradient. Since we are interested in locating critical points rather than in producing a topographic map of the terrain, areas of high gradient contain little information and should be deemphasized.

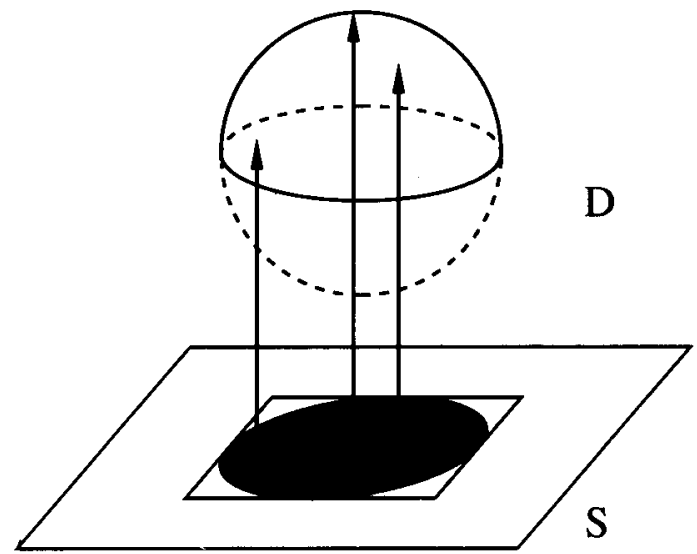

FIG. 2. Inverse orthographic projection from the screen to a spherical phase space. 
The naive choice for the grading function $\mathscr{G}$ is the one adopted by cartographers for producing topographic maps. In such charts, elevations (values of $\mathscr{H}^{*}$ ) map into evenly spaced intervals so as to ensure the following two properties:

$$
\begin{array}{rl}
\forall u, v \in R & u \leqslant v \Rightarrow \mathscr{G}(u) \leqslant \mathscr{G}(v), \\
\forall p, q \in G & \max \left[\mathscr{G}^{-1}(p)\right]-\min \left[\mathscr{G}^{-1}(p)\right] \\
& \approx \max \left[\mathscr{G}^{-1}(q)\right]-\min \left[\mathscr{G}^{-1}(q)\right] .
\end{array}
$$

This simple linear grading scheme, however, ends up highlighting zones of high gradient while washing out areas containing equilibria.

Equidistributed grading ${ }^{7}$ or histogram flattening, ${ }^{8}$ a known image enhancement technique, proves especially effective for making visible the areas of low gradient. This choice for $\mathscr{G}$ constitutes a mapping of $R$ into grades of nearly equal population:

$$
\begin{array}{ll}
\forall u, v \in R & u \leqslant v \Rightarrow \mathscr{G}(u) \leqslant \mathscr{G}(v), \\
\forall p, q \in G & \left|\operatorname{card}\left[\mathscr{G}^{-1}(p)\right]-\operatorname{card}\left[\mathscr{G}^{-1}(q)\right]\right| \leqslant 1
\end{array}
$$

The more points in a given energy band, the finer the detail accentuated by this nonlinear partitioning of the range $R$.

As expected, this scheme works best when the distribution of points in $D$ is independent of the gradient. Furthermore, an equidistributed grading function proves very expensive to compute, since it requires a complete ranking of the values in $R$. For diagrams containing hundreds of thousands of points, this becomes a sizeable computation. Linear grading, on the other hand, requires only the maximum and minimum values of $\mathscr{H}^{*}$, an altogether simpler computation.

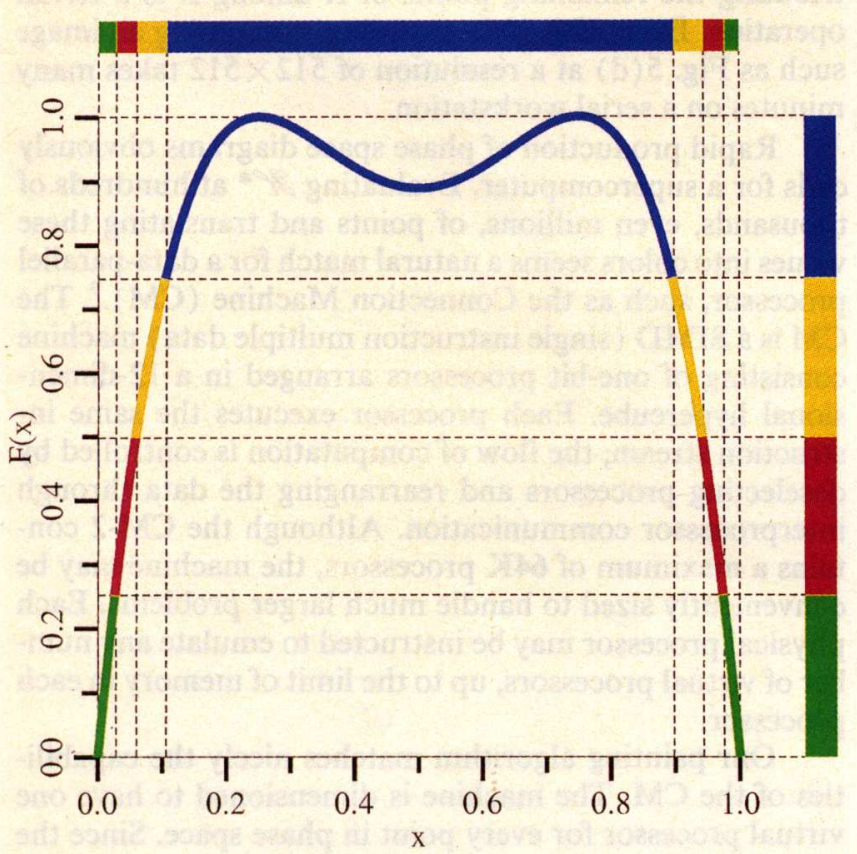

Linear grading
Figure 3 contrasts the two schemes for mapping values of $\mathscr{H}^{*}$ into grades in preparation for display. For purposes of illustration, we graph a one-dimensional quartic having critical points at $1 / 4,1 / 2$, and $3 / 4$, and we assume that only four grades are available (a two-bit color system). As evidenced in the left frame of Fig. 3, the linear grading scheme obscures the local maxima at $1 / 4$ and $3 / 4$. In contrast, the equidistributed grading in the right frame highlights the features of the plateau while ignoring the steep slopes of the function.

\section{GOLOR PALETTES}

Taking advantage of the eye's ability to finely distinguish hues, we select colors for the grades by keeping the intensity and saturation constant and mapping $\mathscr{C}(g)$ to a hue in the interval $C=[0,1)$. To increase contrast, we map $G$ onto $m>1$ spectra. Thus the palette function becomes

$$
\mathscr{C}(\mathrm{g})=(\mathrm{gm} / \mathrm{n}) \bmod 1 .
$$

Taking the same quartic function as before, Fig. 4 demonstrates that increasing $m$ yields finer detail. The left frame shows the grades mapped onto a single spectrum, with resulting loss of detail around the local minimum at $1 / 2$; the right frame shows the same function with $m=4$. Observe the very dense striping around the steep slopes of the function and also the new detail appearing in the basin between $1 / 4$ and $3 / 4$. Too high a value for $m$, however, would have produced an indecipherable striping. Following the ordering of colors indicates the direction of the gradient, and the density of color changes shows the magnitude of the derivative. Note that this coloring scheme assumes no a priori knowledge of the functions; the palette

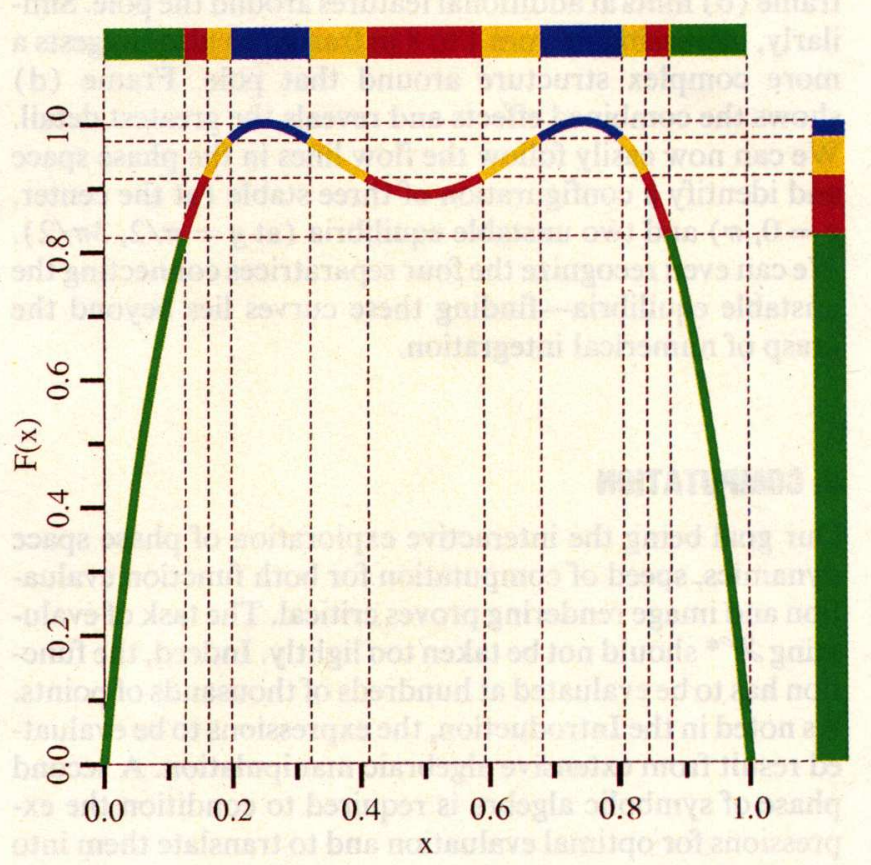

Equidistributed grading

FIG. 3. Two different gradings $\mathscr{G}$ that affect the visibility of equilibria for a simple one-dimensional function. 


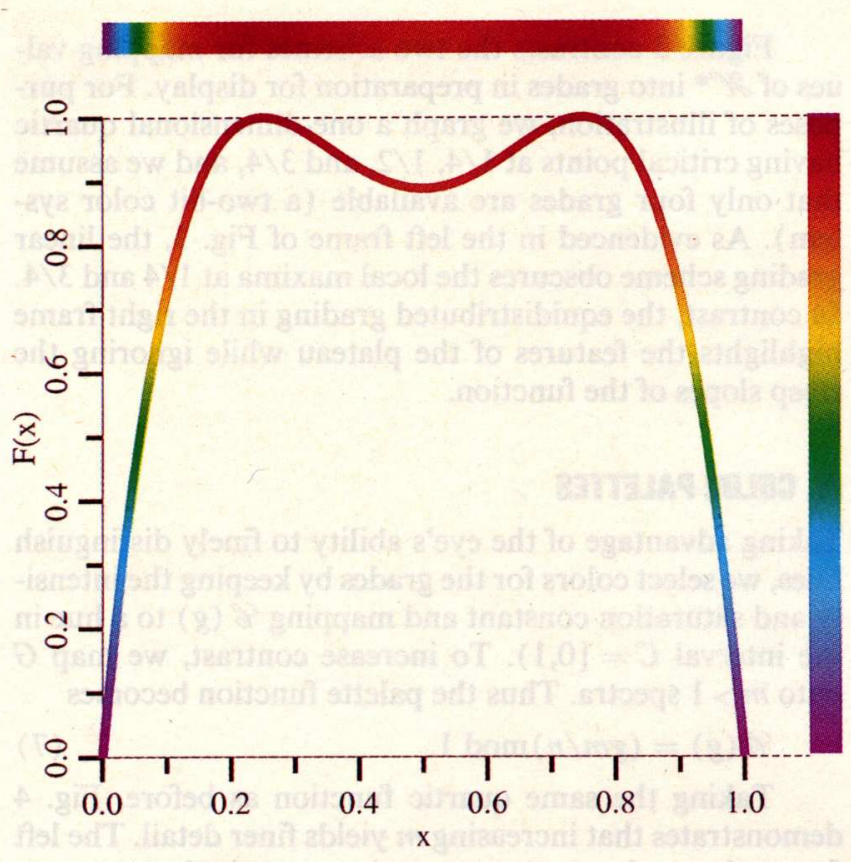

Single spectrum

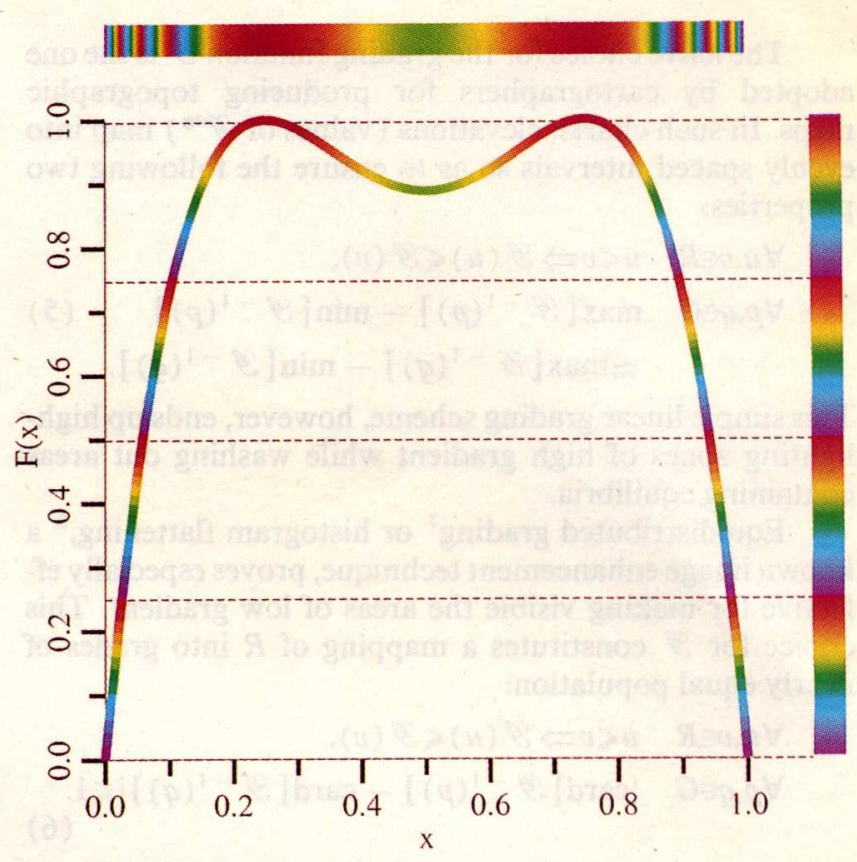

Multiple spectra

FIG. 4. Increasing the number of spectra in the color palette $\mathscr{C}$ improves contrast for the same function illustrated in Fig. 3.

function $\mathscr{C}$ is precomputed to generate the desired striping.

With Fig. 5, we demonstrate the combined effects of demographic grading and color striping. Frame (a) shows the northern hemisphere of phase space for the main problem of satellite theory with linear grading and a single spectrum color map. Switching $\mathscr{G}$ to equidistributed grading in frame (b) hints at additional features around the pole. Similarly, increasing $m$ from 1 to 8 in frame (c) also suggests a more complex structure around that pole. Frame (d) shows the combined effects and reveals the greatest detail. We can now easily follow the flow lines in the phase space and identify a configuration of three stable (at the center, $g=0, \pi$ ) and two unstable equilibria (at $g=\pi / 2,3 \pi / 2$ ). We can even recognize the four separatrices connecting the unstable equilibria-finding these curves lies beyond the grasp of numerical integration.

\section{GOMPUTATION}

Our goal being the interactive exploration of phase space dynamics, speed of computation for both function evaluation and image rendering proves critical. The task of evaluating $\mathscr{H}^{*}$ should not be taken too lightly. Indeed, the function has to be evaluated at hundreds of thousands of points. As noted in the Introduction, the expressions to be evaluated result from extensive algebraic manipulation. A second phase of symbolic algebra is required to condition the expressions for optimal evaluation and to translate them into a form digestible by the graphics routines. Image rendering is also costly; computing the equidistributed grading requires sorting a large population of function values.

Building the equidistributed grading function on a serial machine requires computing time proportional to $p \log p$, where $p$ is the number of points. As an alternative to sorting all the function values, one may approximate the grading function $\mathscr{G}$ by sampling points at regular intervals, say one of every ten, sorting them, and then counting off $n$ partitions to establish the grade boundaries. Incidentally, sampling $R$ may cause the grades to vary significantly in population. Once the grade boundaries have been set, distributing the remaining points of $R$ among $B$ is a trivial operation. Even with $10 \%$ sampling, computing an image such as Fig. $5(\mathrm{~d})$ at a resolution of $512 \times 512$ takes many minutes on a serial workstation.

Rapid production of phase space diagrams obviously calls for a supercomputer. Evaluating $\mathscr{H}^{*}$ at hundreds of thousands, even millions, of points and translating these values into colors seems a natural match for a data-parallel processor, such as the Connection Machine (CM). ${ }^{9}$ The CM is a SIMD (single instruction multiple data) machine consisting of one-bit processors arranged in a 12-dimensional hypercube. Each processor executes the same instruction stream; the flow of computation is controlled by deselecting processors and rearranging the data through interprocessor communication. Although the CM-2 contains a maximum of $64 \mathrm{~K}$ processors, the machine may be conveniently sized to handle much larger problems. Each physical processor may be instructed to emulate any number of virtual processors, up to the limit of memory in each processor.

Our painting algorithm matches nicely the capabilities of the CM. The machine is dimensioned to have one virtual processor for every point in phase space. Since the energy function is the same at each point in $D$, the CM evaluates $\mathscr{H}^{*}$ all at once. Since the evaluation at any point is independent of its neighbors, no interprocessor communication is required-a relatively time consuming operation on the CM. Of course on the connection machine, we 


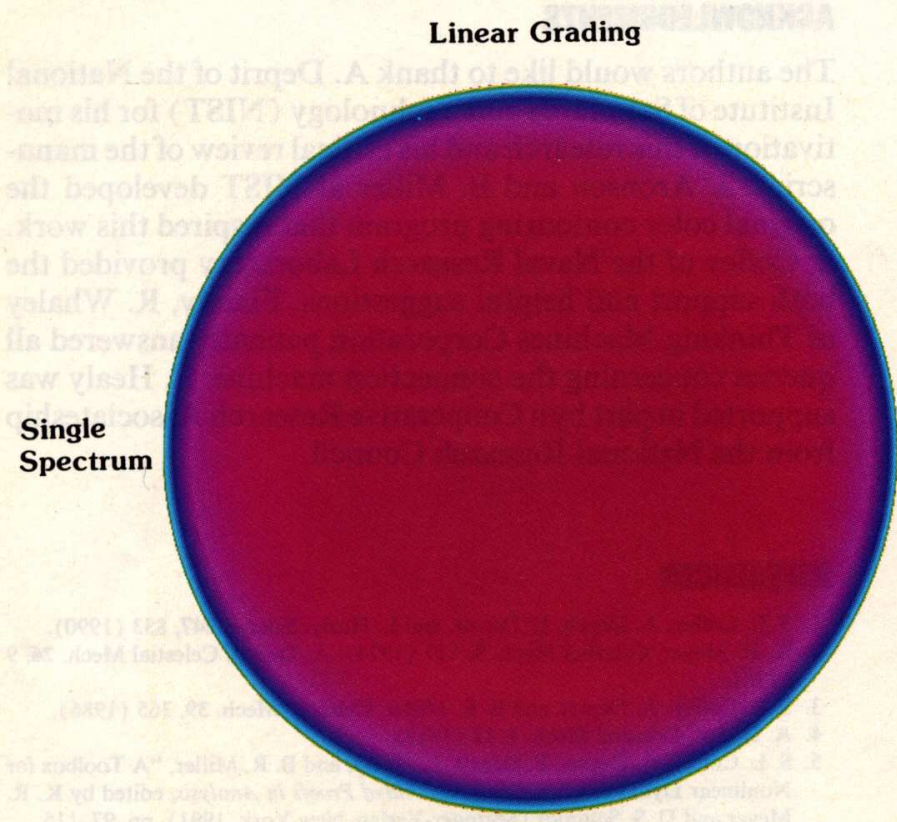

(a)

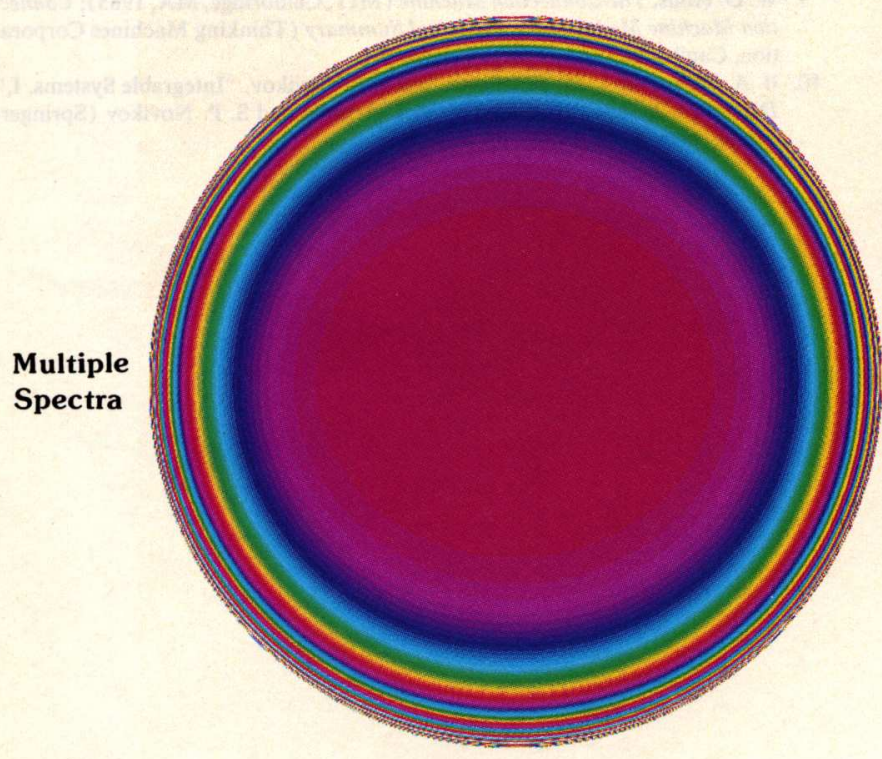

(c)
Equidistributed Grading

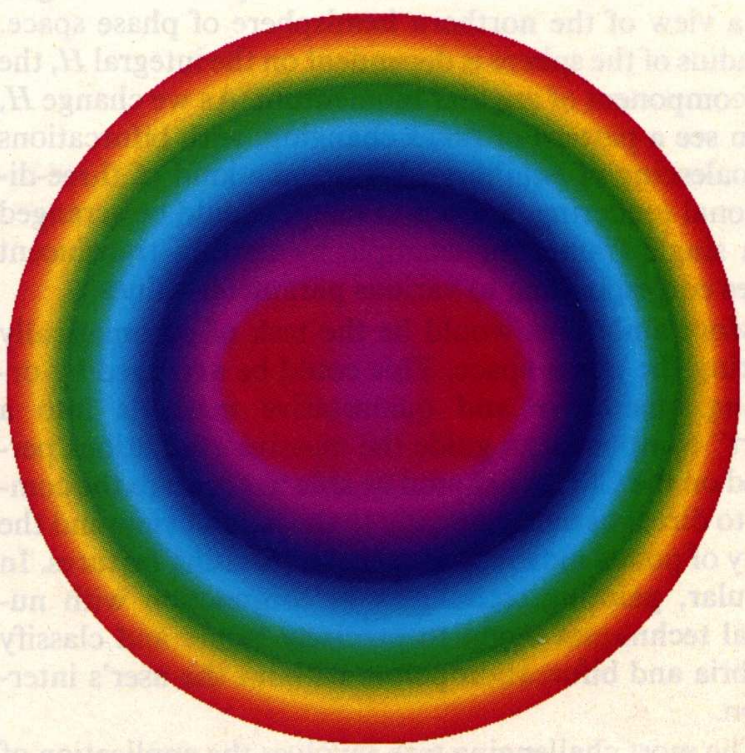

(b)

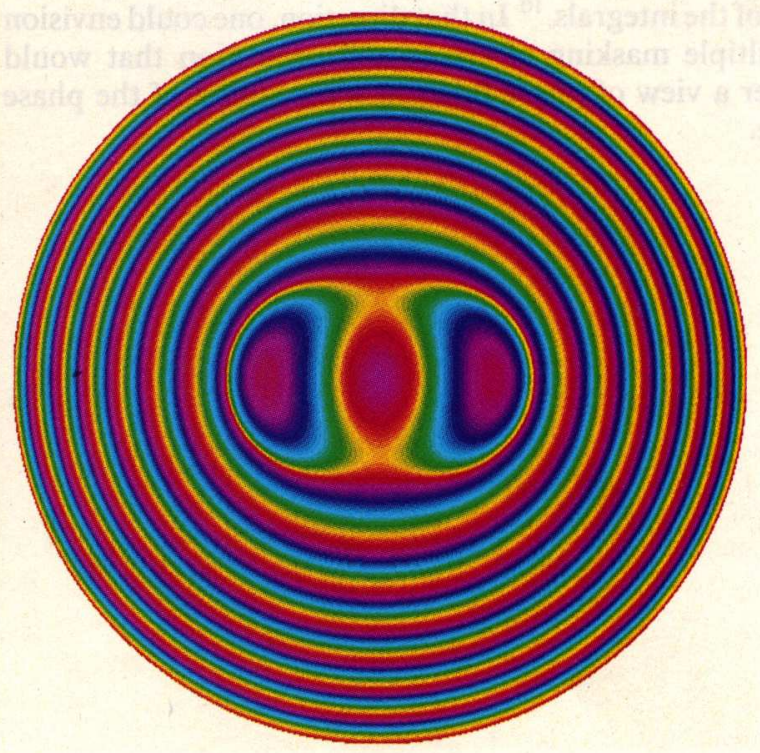

(d)

FIG. 5. The northern hemisphere of phase space rendered with four combinations of gradings and color palettes.

do not need sampling to establish the equidistributed grading function, because we can afford to sort all the energies and to enumerate them by unique values. After completing the ranking, we assign approximately equal numbers of unique values to each grade in ascending order. The CM software ranks values by mapping a binary tree onto the hypercube network, and the sorting of $R$ for the equidistributed grading becomes an $\mathscr{O}(\log p)$ operation. For the main problem of artificial satellite theory, producing images such as Fig. 5 (d) on a $16 \mathrm{~K}$ connection machine takes about one second, with most of the time spent in the grading algorithm rather than in the Hamiltonian evaluation.

\section{FUTURE}

Our future work on this technique will proceed in three directions: improvements to the current method, development of algorithms to automate the search for equilibria, and application to Hamiltonian systems of higher dimensions.

We would like to make the image rendering sophisticated. This could be accomplished by using the CM simply as a computational engine to evaluate a large set of points in phase space, rather than using the inverse projection $\mathscr{P}$ to determine the points at which to compute. The dataset would then be rendered on a graphics workstation 
equipped with the rendering tools currently lacking on the Connection Machine. For instance, the pictures in Fig. 5 show a view of the northern hemisphere of phase space. The radius of the sphere is dependent on the integral $H$, the polar component of angular momentum. As we change $H$, we can see a pattern of flows changing, with bifurcations and coalescing of equilibria. With some kind of three-dimensional rendering, a series of images could be arranged into a single frame, for example by nesting translucent spheres corresponding to various parameter values.

More ambitious would be the task of automatically exploring the phase space. This could be arranged by organizing qualitative and quantitative methods into a "smart" component to guide the massive evaluations performed on the CM. We should be able to program the computer to direct us automatically to the field of view and the density of points more apt to reveal interesting features. In particular, painting techniques in conjunction with numerical techniques ought to precisely locate and classify equilibria and bifurcation points without the user's intervention.

The most challenging task involves the application of "painting" to Hamiltonians of higher degree. If there are $n$ known integrals of an $n$ degree of freedom system, the flows can be identified as the intersections of the level surfaces of each of the integrals. ${ }^{10}$ In that direction, one could envision a multiple masking of colors and projection that would render a view of phase flows in some subset of the phase space.

\section{ABKNOWLEDENENTS}

The authors would like to thank A. Deprit of the National Institute of Standards and Technology (NIST) for his motivation of this research and his critical review of the manuscript. J. Aronson and B. Miller at NIST developed the original color contouring program that inspired this work. S. Coffey of the Naval Research Laboratory provided the both support and helpful suggestions. Finally, R. Whaley of Thinking Machines Corporation patiently answered all queries concerning the connection machine. L. Healy was supported in part by a Cooperative Research Associateship from the National Research Council.

\section{REFERENCES}

1. S. L. Coffey, A. Deprit, E. Deprit, and L. Healy, Science 247, 833 (1990).

2. K. R. Meyer, Celestial Mech. 9, 517 (1974); A. Deprit, Celestial Mech. 26, 9 (1982).

3. S. L. Coffey, A. Deprit, and B. R. Miller, Celestial Mech. 39, 365 (1986).

4. A. Deprit, Celestial Mech. 1, 12 (1969).

5. S. L. Coffey, A. Deprit, E. Deprit, L. Healy, and B. R. Miller, "A Toolbox for Nonlinear Dynamics," in Computer Aided Proofs in Analysis, edited by K. R. Meyer and D. S. Schmidt (Springer-Verlag, New York, 1991), pp. 97-115.

6. A. Deprit, J. Guid. Control 4, 201 (1981).

7. J. Kautsky and N. K. Nichols, SIAM J. Sci. Stat. Comput. 3, 145 (1982)

8. R. Hummel, Comput. Graph. Image Process. 4, 209 ( 1975); R. Hummel, Comput. Graph. Image Process. 6, 184 (1977)

9. W. D. Hillis, The Connection Machine (MIT, Cambridge, MA, 1985); Connection Machine Model CM-2 Technical Summary (Thinking Machines Corporation, Cambridge, MA, 1987), Version 5.1.

10. B. A. Dubrovin, I. M. Krichever, and S. P. Novikov, "Integrable Systems. I," Dynamical Systems IV, edited by V. I. Arnol'd and S. P. Novikov (SpringerVerlag, New York, 1990), pp. 173-280. 